\title{
Throughput Capacity of Hybrid Multi-channel Vehicular Ad Hoc Networks
}

\author{
Chen Lin* \\ Department of Computer Application and Engineering, Shanghai Second Polytechnic University, \\ Shanghai, China 201209
}

Keywords: Capacity; Vehicular Ad hoc Networks; Hybrid Multi-channel; Mobility

\begin{abstract}
The throughput capacity of hybrid multi-channel Vehicular Ad hoc Networks is addressed in the paper. Both the theoretic upper and lower bounds are obtained. Moreover, both single road and grid road are analyzed. The results show that the movement has little influence on the throughput capacity of hybrid multi-channel vehicular ad hoc networks.
\end{abstract}

\section{Introduction}

A basic attribute of Wireless network is the asymptotic capacity which reflects the network performance changing rule with increasing network size[1]. Furthermore, the analysis and evaluation of wireless network is the fundamental base to plan and design the network. The network capacity is constrained by multiple factors, such as flow model, bandwidth, energy and network topology.In the landmark paper[2] Gupta and Kumar presented that when there are $\mathrm{n}$ nodes uniformly distributed in an unit circle, and each node randomly selects another nodes as its destination, then the capacity of each node is $\lambda(n)=\Theta(W / \sqrt{n \log n})$. This is a disappointing result, for it shows that the large scale of ad hoc network is infeasible.

One way to improve the network capacity is to adopt multi-channel technology. For the general ad hoc networks, Kyasanur and Vaidya[3] studied the relation between the number of channels and that of interfaces of each nodes. In the model, the interfaces can switch freely among channels. The analysis results show that for stochastic network, when the ratio between the number of channels to that of interfaces is in the interval $\mathrm{O}(\log n)$, there is no capacity loss; however, if the ratio is greater than the interval, then there has been capacity loss.

Add base stations, building hybrid network is another way, and it is more feasible. Liu et al.[4] studied hybrid wireless network model which assumes base stations are fixed in the hexagon lattices, and ad hoc nodes are randomly distributed among the whole area. The results showed that when there are ${ }^{m}$ base stations and $n$ ad hoc nodes in the network, and the growing rate of $m$ is less than $\sqrt{n}$, the network capacity improves little; when the growing rate is greater than $\sqrt{n}$, it increases linearly with the growth of $m$. Kozat and Tassiulas studied the stochastic hybrid network, where both base stations and ad hoc nodes are randomly distributed in the unit area, and the ratio of the number of base stations to ad hoc nodes is constant, then the capacity of each ad hoc node is $\Theta(W / \log n)$. Kozat and Tassiulat[5] studied the network capacity where the growth rate of the number of base stations $m$ is located at different intervals.

As one kind of special Ad hoc network, Vehicular Ad hoc Networks (VANET) is an open and mobile Ad hoc network where vehicles on road can communicate with each other and with roadside access points [6]. It has similar features with other Ad hoc networks such as multi-hops, dynamic topology. However, it also has own characters. For example, vehicles usually run on road, and are impossible distributed randomly on areas; energy shortage may not be a critical issue for VANET; VANET may support multiple channels with multiple interfaces. 
Due to its significant potential application ability, VANET has attracted great research focus. But the throughput capacity of VANET is still in its infancy[7]. Its complex mobility and topology mechanism lead to difficulties to model and make quantitative analysis about its throughput capacity. Although [7] investigated throughput scaling laws for VANET, the impact on capacity based multi-channel technology had not been dealt with.

In the paper, the throughput capacity of hybrid multi-channel VANET is investigated. Two scenes of road topology are analyzed. Furthermore, the upper and lower bounds are both obtained. The rest of the paper is organized as follows: section 2 embodies the definitions and notations; section 3 is dedicated to the study of throughput capacity for VANET with single road; downtown grid is dealt with in section 4 . The paper is finally concluded in section 5 .

\section{Definition and notation}

We use the following notation to represent asymptotic bounds:

$$
\begin{aligned}
& f(n)=\mathrm{O}(g(n)) \text { means there exists some constant } \alpha \text { and integer } N \text { such that } \alpha \text { for } n>N . \\
& f(n)=o(g(n)) \text { means that } \lim _{n \rightarrow \infty} f(n) / g(n)=0 \\
& f(n)=\Omega(g(n)) \text { means that } g(n)=\mathrm{O}(f(n)) \\
& f(n)=\omega(g(n)) \text { means that } g(n)=o(f(n)) \\
& f(n)=\Theta(g(n)) \text { means that } f(n)=\mathrm{O}(g(n)) \text { and } g(n)=\mathrm{O}(f(n))
\end{aligned}
$$

The traffic between a source-destination pair is referred to as a "flow". As in [2], we say that per flow capacity is $\lambda$ if each flow in the network can be guaranteed a throughput of at least $\lambda$, and the network capacity is defined to the aggregate throughput over all the flows in the network, $n \lambda$

\section{Model}

The network model is the same as [7]. A lane on road is parameterized a smooth continuous curve $X_{n}(s)=\left(x_{n}(s), y_{n}(s)\right), s \in[0,1]$ on the plane. There are $n$ vehicles on the road, and the road length is $L$ and the density $k_{n}(s)$ is defined by the average number of vehicles per unit length at point $X_{n}(s)$ which is assumed to be a bounded positive number. We also assume $k_{n}(s)=k$, for all $s \in[0,1]$ in all proofs.

The mobility model for vehicle is assumed at a Poisson process which is similar as [7]. At time zero, all vehicles on the same lane will choose a common speed $v \in\left[0, v_{\max }\right]$ uniformly at random.

These vehicles are capable of transmitting and receiving $W^{\text {bits/s via all }}{ }^{c}$ channels, and the base station nodes can also communicate with vehicles by these common channels. Any nodes including vehicles and base stations is equipped with $m$ interfaces where $1 \leq m \leq c$, and each interface is assumed to be able to communicate on any channel. Similar to, we use notation $(m . c)$ network to refer to network with $m$ interfaces per node and ${ }^{c}$ channels.

\section{Throughput capacity for hybrid multi-channel VANET with Single Road}

We divided the road $L$ into $\frac{L}{\ln n}$ segments with each segment length of $\ln n$, and each segment is deployed one base station which is shown in Fig. 1. Each node has a communication radius $r=\ln n$. 


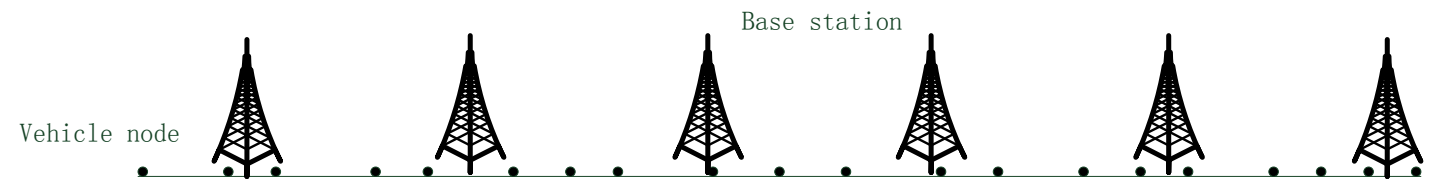

Fig. 1 Single Road

Upper bound. The throughput capacity of hybrid multi-channel VANET is also limited by two constraints, each of them is used to obtain a bound on the network capacity. The minimum of the two bounds is an upper bound.

As stated in [7], the topology changes caused by vehicle movement is slow which has few impact on its capacity.

Constraints 1 Interference constraints: It can be shown from[7] that the throughput capacity of hybrid multi-channel VANET is $\mathrm{O}\left(\frac{n W}{\ln n}\right)$ bits/s. The upper bound is limited by interference, and the bound for hybrid VANET with single interface is also applicable to hybrid multi-channel VANET as well.

Constraints 2 Interface constraints: The capacity of a multi- channel wireless network is also limited by the number of simultaneous transmissions supported by interfaces in the network. According to the channel model, each interface can transmit at a rate of $\frac{W}{c}$ bits/s, and each node has $m$ interfaces, so the total network capacity is bounded by $\mathrm{O}\left(\frac{n W m}{c}\right)_{\mathrm{bits} / \mathrm{s}}$

Combined with the two bounds, the network capacity is bounded by $\mathrm{O}\left(\min \left(\frac{n W m}{c}, \frac{n W}{\ln n}\right)\right)$ bits/s. Thus, the following theorem is obtained:

Lemma 1. The upper bound of throughput capacity of hybrid multi-channel VANET is as follows:

When $\frac{c}{m}$ is $\mathrm{O}(\ln n)$, network capacity is $\mathrm{O}\left(\frac{n W}{\ln n}\right)$ bits/s

When $\frac{c}{m}$ is $\Omega(\ln n)$, network capacity is $\mathrm{O}\left(\frac{n W m}{c}\right)$ bits/s

Lower bound. In this section, we provide a construction of the routing scheme and scheduling strategy to establish a lower bound on the network capacity. We will first provide a construction of a routing scheme and a transmission schedule for $\mathrm{a}(1, \mathrm{c})$ network, and then extend the result to $\mathrm{a}(\mathrm{m}, \mathrm{c})$ network by using Lemma 2 in [7].

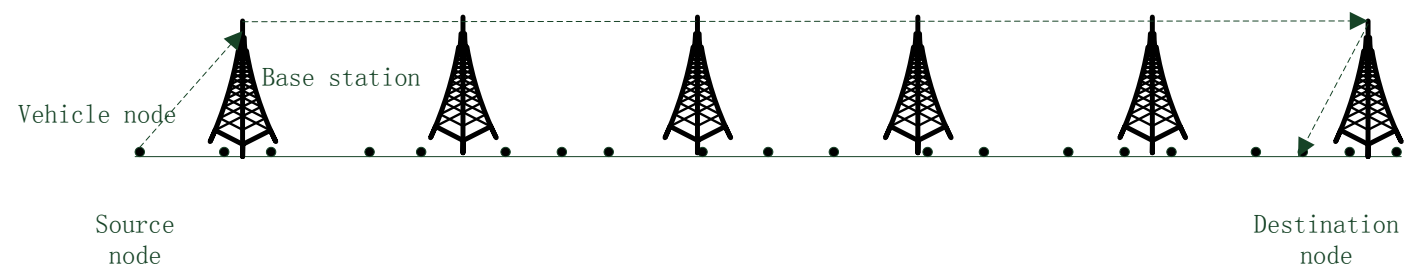

Fig. 2 Routing Scheme

The routing scheme is shown in Fig. 2. The source vehicle node sends packets to the nearest base station, and then the base station forwards them to that base station nearest to its destination vehicle through additional communication link such as high speed wired transmission. 
Lemma 2. The number of segments that interfere with the transmission of a specific segment is bounded above by a constant.

A standard graph coloring method can be used to prove the lemma. It can be shown that we can schedule parallel transmissions in the network[8].

Theorem 1 . The throughput capacity of hybrid multi-channel VANET with Single Road is as follows:

When $\frac{c}{m}$ is $\mathrm{O}(\ln n)$, network capacity is $\Theta\left(\frac{n W}{\ln n}\right)$ bits/s

When $\frac{c}{m}$ is $\Omega(\ln n)$, network capacity is $\Theta\left(\frac{n W m}{c}\right)$ bits/s

The proof process is similar to that in [8-9]. We averagely assign transmissions of vehicle nodes on any segment to $c$ channels. Then It is evident that the number of transmissions in any segment is $\mathrm{O}\left(\frac{\ln n}{c}\right)$. We can construct a conflict graph: each transmission is a separate vertex, and the edge connecting two vertexes should satisfy two requirements that have the same channel or have at least one same endpoint. Last, according to the vertex-coloring the theorem can be proved with standard process.

\section{Throughput capacity for hybrid multi-channel VANET with Grid}

The road grid is usually downtown topology which is shown in Fig. 3. The grid should satisfy the requirements of sparse conditions which is detailed in [7][10].

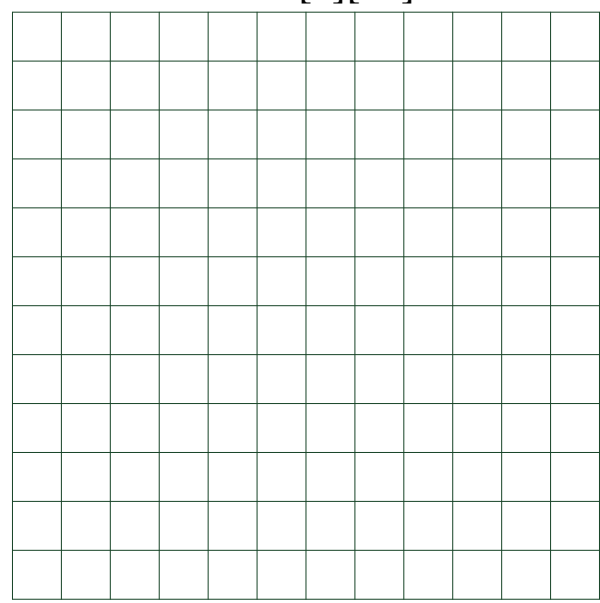

Fig. 3 Downtown topology

Theorem 2. The throughput capacity of hybrid multi-channel VANET with Grid Road is as follows:

When $\frac{c}{m}$ is $\mathrm{O}(\ln n)$, and road sparse condition is satisfied, then network capacity is $\Theta\left(\frac{n W}{\ln n}\right)$ bits/s

When $\frac{c}{m}$ is $\Omega(\ln n)$, and road sparse condition is satisfied ,then network capacity is $\Theta\left(\frac{n W m}{c}\right)$ bits/s

It can be shown from the proof process of theorem 1 that road topology does not have impact on throughput capacity for hybrid multi-channel VANET. It is different from normal VANET. Because with the help of base stations, the road topology limits on throughput capacity of hybrid multi-channel VANET is removed. 


\section{Conclusions}

In the paper, the throughput capacity of hybrid multi-channel VANET is dealt with. The theoretic upper and lower bounds is obtained. The analysis process shows that the movement of vehicles has no impact on hybrid multi-channel VANET. Furthermore, the ratio of interfaces to channels has great influence on throughput capacity.

\section{Acknowledgement}

This research was supported by National Natural Science Foundation of China (Grant No. 61202368), Shanghai Natural Science Foundation of China (Grant No. 12ZR1411500) and Innovation Program of Shanghai Municipal Education Commission (Grant No. 13ZZ141).

\section{References}

[1] L. Chen, S.T. Wei. Throughput capacity of hybrid multi-channel wireless networks. AEU-International Journal of Electronics and Communications, 64(2010), No.4, 299-303

[2] P. Gupta, P.R. Kumar. The capacity of wireless networks. IEEE Transactions on Information Theory, 46(2000), 388-404

[3] P. Kyasanur, N. Vaidya. Capacity of multi-channel wireless network: impact of number of channels and interfaces. Proceedings of ACM mobicom, Gologne, Germany(2005)43-57

[4] B. Liu, Z. Liu, D. Towsley. On the capacity of hybrid wireless networks. Proceedings of IEEE Infocom, San Francisco, CA, USA(2003) 1543-1552

[5] U. Kozat, L. Tassiulat. Throughput capacity of random ad hoc networks with infrastructure support. Proceedings of ACM mobicom, San Diego, CA, USA(2003)55-65

[6] L.J.Chen, H.Jiang, J.Wu et al. Research on Transmission Control on Vehicle Ad-Hoc Networks. Journal of Software, 18(2007): 1477-1490

[7] M.Nekoui and H. Pishro-Nik. Throughput Scaling laws for Vehicular Ad Hoc Networks. IEEE Transactions on Wireless Communications, Vol.11,No.8(2012):2895-2905.

[8] V. Bhandari, N. Vaidya. Connectivity and Capacity of multi-channel wireless networks with channel switching constraints. Technical Report, University of Illinois at Urbana-Champaign, USA, 2006

[9] L.Chen, S.T.Wei and W.A.Tan. Network Capacity of Multi-channel Multi-interface Hybrid Networks. Computer Science, Vol.38, No.8(2011): 96-100.

[10] M.Nekoui, A.Eslami and H. Pishro-Nik. The capacity of Vehicular Ad Hoc Networks with infrastructure. Proceedings of 2008 Modeling and Optimization in Mobile, Ad Hoc, and Wireless Networks and Workshops(2008): 267-272. 